\title{
Prevalence and Predictors of Traditional, Complementary/Alternative Medicine use, and types of herbal remedies used for COVID-19 in the South West Region of Cameroon
}

\section{Charlotte Wenzi Ayima}

Department of Public Health and Hygiene, Faculty of Health Sciences, University of Buea

\section{Nana Theophile Njamen}

University of Buea

Assob Jules Clement Nguedia

University of Buea Faculty of Health Sciences

Dickson Shey Nsagha ( $\square$ nsaghads@hotmail.com )

Department of Public Health and Hygiene, Faculty of Health Sciences, University of Buea, P.O Box 12, Buea

\section{Research Article}

Keywords: Prevalence, Predictors, TCAM, COVID-19, South west Region, Cameroon

Posted Date: August 30th, 2021

DOI: https://doi.org/10.21203/rs.3.rs-847310/v1

License: (1) This work is licensed under a Creative Commons Attribution 4.0 International License. Read Full License 


\section{Abstract \\ Background}

Up to the 21 st century, traditional, complementary and alternative medicine(TCAM) is still used despite the wide spread of orthodox medicine. The magnitude, predictors and types of herbal remedies used for COVID-19 are assessed to tailor evidence based policy and drug formulation against COVID-19.

\section{Methods}

A community based cross sectional study was conducted among 1100 respondents from nine (9) communities selected in three health districts from May to July 2020. Pretested structured questionnaire were used to collect data from selected households on the use of TCAM, predictors as well as on the herbal preparations used for COVID-19. Nineteen (19) traditional healers were also interviewed on the types of herbal preparations use on patients tested or suspected for COVID-19 and the signs and symptoms identified. P-value $<0.05$ were considered to be statistically significant in multivariate logistic regression analysis. Atlas ti $\vee 7.5$ was used for types of herbal species reported.

\section{Results}

Of the 1100 participants sampled, 754 (68.5\%) had used TCAM in the last 12 months, $95 \% \mathrm{Cl}$ : $66.3-71.5$. A total of $24.4 \%$ [95\%Cl: $20.6-$ 29.1] of the participants used herbal remedies for COVID-19. Allium sativum, Azadirachta indica, Zingiber officinale, Artemisia annua were most commonly used herb for COVID-19. Cough, catarrh and fever were the main symptoms of COVID-19. Herbalists $79.7 \%$ [95\% Cl: 77.3-82.1] and bone setters $14.9 \%$ [95\% Cl: $12.8-17.0$ ] were mostly visited. Logistic regression analysis showed age $>41$ years $(95 \% \mathrm{Cl}$ : 1.09-4.91), being a farmer (95\% Cl: 1.99-5.34), income levels between 185-370 USD (95\% Cl: 1.33-4.55), participants who resided in a rural setting (95\% Cl: 1.04-3.98), being knowledgeable on TCAM (95\% Cl: 1.54-6.45) and having a positive attitude towards TCAM (95\% Cl: 1.94-6.45) were predictors for TCAM use.

\section{Conclusion}

TCAM is widely used even in the era of orthodox medicine, and many factors contribute to its use in the Southwest region of Cameroon which should be taken into consideration in healthcare interventions that are sensitive to TCAM. Herbal preparations used during the COVID-19 pandemic can serve as baseline for drug development through efficacy and toxicity tests.

\section{Introduction}

Traditional, complementary and alternative medicine (TCAM) are a group of healthcare practices (indigenous or imported) that are delivered outside of the mainstream healthcare system [1]. In the African context TCAM may include the use of local herbal medicines or products, indigenous healthcare practices (such as traditional bone setting, traditional birth attendants), as well as imported complementary and alternative medicine products and practices (eg, acupuncture or chiropractic) [2]. According to the world health organization, TCAM can contribute to improving quality of healthcare services through integration, regulation of TCAM products/services used by communities, strengthening sustainability and resilience through maximizing potentials of TCAM [3]. WHO estimates that more than $80 \%$ of the African populations rely on traditional medicine for their healthcare needs [4]. A systematic survey from 32 countries from non-Sub-Saharan Africa region shows a TCAM provider use prevalence of $34.7 \%$ in Australia, in Europe ranging from under $10 \%$ in Bulgaria, Poland and Slovenia to $35.4 \%$ in France, in Asia from 16.7\% in Russia, to over 50\% in China mainland, the Philippines and Republic of Korea, and over $20 \%$ in the USA, Chile and South Africa [5]. A recent systematic survey conducted in SSA shows traditional, complementary and alternative medicine use in the general population, reporting substantial prevalence ranging from $4.6 \%$ (urban settlement in Ethiopia) to $94 \%$ (semi urban settlements in Nigeria and Ethiopia), with an estimated average of 58.2\% [6]. Various prevalence was also observed among 10 articles that reported on TCAM practitioner utilization (1.2-67\% (mean, 28.8\%)) across four identified studies with smaller sample sizes. The with higher prevalence reported in Tanzania (77.1\%) [6]. Reports from world health organization show TCAM use in Uganda and Tanzania at 60\%, in Benin and Rwanda at 70\%, and in Ethiopia at 90\% [6]. 
In low- and middle-income countries, TCAM use has been associated with lower educational and socio-economic status[7, 8], relative low cost and flexibility of payment of TCAM products and services, accessibility, perception of TCAM being natural and therefore safe as well as effective compared with orthodox healthcare. In another study patient's trust and confidence in their traditional medicine practitioners were factors associated with TCAM product and practitioner use[6].

Unsurprisingly, there has been an uptick in the amount of research being conducted at the intersection of TCAM and COVID-19[9]. Several doctors and researchers have already attempted to use herbal medicines on clinical trials against SARS-CoV-2[9].The longstanding use of dietary therapy and herbal medicine to prevent and treat diseases cannot be overemphasized, as several herbs exhibit antiviral activity[10]. Types of herbal medicine to prevent SARS-CoV-2 infections could serve as a baseline for drugs development[11].

Recently the Madagascar Institute of Applied research linked the use of Artemisia annua (sweet wormwood) in COVID-19[11]. Zimbabwean government was reported to have authorized herbalists to treat patients with COVID-19 symptoms raising concerns among national public health experts [9]. There is a lack of adequate data on the use of TCAM during the COVID-19 pandemic in Africa and in Cameroon in particular.

There is dearth of research and data from Cameroon on the extend and predictors of TCAM use, as well as types of herbal preparations use in the general population for COVID-19 which is very necessary for policy formulation/population based healthcare interventions that are sensitive to TCAM, regulation and further research on the efficacy and toxicity of these herbals use for COVID-19.

\section{Materials And Methods}

\section{Study Area}

Cameroon has about $90 \%$ of the African ecosystems which includes; the Sahelian, Sudan, humid tropical forest, afro mountains, coastal and mountain eco-regions [12]. This study was conducted in the Fako Division in the Southwest Region of Cameroon. Fako Division lies in the coastal region between $4^{\circ} 28^{\prime} 30^{\prime \prime} \mathrm{N}$ and $3^{\circ} 54^{\prime} 26^{\prime \prime} \mathrm{N}$ latitudes, and $8^{\circ} 57^{\prime} 10^{\prime \prime} \mathrm{E}$ and $9^{\circ} 30^{\prime} 49^{\prime \prime} \mathrm{E}$ longitudes. The land area is approximately $203,071.27$ ha and has a population of 534854 people. Most inhabitants practice agriculture as the main economic activity [12]. The region has two seasons: the dry season from October to March and the wet season from April to September. Almost all ethnic groups in Cameroon are represented in the region, attracted by the fertile volcanic soil for agriculture. The study was conducted in nine (9) communities (Isokolo-Limbe, Mile 4-Limbe, Down Beach-Limbe, Buea town, Muea-Buea, Mile 16-Buea, Likumba-Tiko, Water tankTiko(Camp) and Small Ekange-Tiko (Camp) i.e 3 communities selected from the Buea, Tiko, and Limbe Health Districts in the Southwest Region of Cameroon.

\section{Study Design}

This was a cross-sectional survey conducted in 9 communities in the South west region Cameroon. Nine communities were selected within the 3 health districts and semi-structured questionnaires administered to examine traditional, complementary and alternative medicine use from May to July 2020. In the nine communities' traditional healers were also interviewed on the type of herbal preparations used for COVID-19, mode of preparation and also on the signs and symptoms of COVID-19.

\section{Study Population}

The population of the study included households from the nine communities. The study population included individual from the age of 21years and above who were living for not less than six months in the community. The sampling units were households, while the study units were adult individual available in the household during the interview. The study population also included traditional healers residing in the communities.

\section{Sample Size Determination}

The Cochran formula was used to determine the minimum sample size required in this study. It allows for calculation of the best possible size with preferred precision and confidence level.

Sample size was calculated based on the prevalence of TCAM use following assumptions: $P=50 \%$ (prevalence of TCAM use) 


$$
\mathbf{n}=\frac{(\mathrm{z})^{2} \alpha \mathbf{p}(\mathbf{1}-\mathbf{p}) d}{\mathbf{i}^{2}}
$$

$n=$ sample size $\quad Z a=$ constant $($ Confidence interval, $95 \%)=1.96$ at $a=0.05, d=2.8$ correction factor, $i=$ Precision of the event of interest (degree of accuracy) $=0.05$

$p$ (population prevalence $)=50 \%$. The prevalence of TCAM use in the Fako Division is unknown, therefore maximum heterogeneity was assumed (ie, a 50/50 split in users and non-users) with $p=0.5$

$$
\mathbf{n}=\frac{(1.96)^{2} \alpha 0.5(1-0.5) 2.8}{0.05^{2}}
$$

\section{8 participants}

Considering a $2 \%$ non-response rate, a total of 1100 respondents were included in this study. Probability proportionate to size was used to determine the number of respondents that were recruited from each health district.

\section{Sampling Procedure}

Systematic random sampling technique was used to select households in the nine communities. The first household per community was selected from the list of initial $\mathrm{n}$ households by balloting method. Then every $\mathrm{nth}$ household was selected and an adult in the household was interviewed. In the presence of more than one adult, balloting was done to choose one adult who was interviewed. Traditional healers were purposively selected to take part in the interview sessions from the study communities to assess herbal remedies used for COVID-19, mode of preparationas well as the signs and symptoms.

\begin{tabular}{|c|c|c|c|}
\hline Health district & Total adult Population & Proportionate sample per district & \\
\hline Buea & 173,526 & 360 & $\begin{array}{l}\text { population of community/Total adult population of the } \\
\text { 3communities per district) } \times \mathrm{n} \text { (sample size per district) }\end{array}$ \\
\hline Tiko & 154,690 & 320 & \\
\hline Limbe & 202,831 & 420 & \\
\hline Total & 531,047 & 1100 & $\begin{array}{l}\text { Table 2: Sample size per study site based on probability } \\
\text { proportionate to size among Community participants }\end{array}$ \\
\hline
\end{tabular}

\section{Table 1: Sample size per health district based on probability proportionate to size}

\begin{tabular}{|lll|}
\hline Study site & Total adult Population & Number of participants per site (Proportionate sample) \\
\hline Buea Town & 15,272 & $138(38.3 \%)$ \\
\hline Muea & 13,161 & $119(33.3 \%)$ \\
\hline Mile 16 & 11,321 & $103(29.0 \%)$ \\
\hline Total & 39,754 & $360(100 \%)$ \\
\hline Water Tank & 2,338 & $136(42.4 \%)$ \\
\hline Likumba & 2,743 & $159(49.7 \%)$ \\
\hline Small Ekange & 428 & $25(8.0 \%)$ \\
\hline Total & $\mathbf{5 5 0 9}$ & $\mathbf{3 2 0}(\mathbf{1 0 0})$ \\
\hline Mile 4 & 25,859 & $360(85.7 \%)$ \\
\hline Isokolo & 812 & $12(3.0 \%)$ \\
\hline Down Beach & 3,469 & $48(11.5 \%)$ \\
\hline Total & $\mathbf{3 0 , 1 4 0}$ & $\mathbf{4 2 0}(\mathbf{1 0 0})$ \\
\hline
\end{tabular}




\section{Data Collection Procedures}

Data was collected by trained data collectors using pre-tested semi-structured questionnaires through face-to face interview. Structured closed and open ended questionnaire was adapted from standardized questionnaires such as the I-CAM (International questionnaire for CAM use) [13] and published articles in peer-reviewed journals. The questionnaire was divided into three sections: first section dealt with respondent's information on the socio-demographic characteristics, the second section dealt with the use of TCAM in the past 12months and for various ailments while the third section captured information on the community use of TCAM against COVID-19 and other ailments. Out of the 1119 copies of the questionnaire distributed, 1100 were returned in the community-based survey, giving a response rate of $98 \%$. Interview guides were designed to capture information of the types of herbal preparation used by traditional healers for COVID-19 patients as well as the signs and symptoms for COVID-19.

\section{Data Quality Control}

Trained data collectors (postgraduate and undergraduate students) were used for the data collection process. The training involved a systematic and comprehensive explanation of the research process through extensive discussions. Included in these were questions and answers and demonstrations. The training also enabled the data collectors to acquaint themselves with field operations techniques. Pretesting was done to validate consistency of the questions and data collection tool.

\section{Study Variables}

The outcome variables of the study were prevalence of TCAM use and types of herbal remedies used for COVID-19 in the study area. The explanatory variables were age, location, monthly family income, educational status, religion, and ethnicity.

\section{Data Management and Analysis}

Questionnaires from the field were cross checked each time they were brought for unfilled and unanswered questions. Coding was also checked to ensure the use of correct codes by the principal investigator. Data was entered into excel, cleaned, and analyzed using SPSS Version 25 (Statistical Package for the Social Sciences) for Windows. The results were presented using simple frequencies with percentages in appropriate tables to display the descriptive part of the result. Multivariate logistic regression was used to determine the predictors associated with TCAM use. ATLAS.ti 7.5 (Scientific Software Development GmbH, Berlin) was used to code and analyze types of herbal preparations used for COVID-19 as well as the signs and symptoms. All statistical tests were performed using two-sided tests at the 0.05 level of significance.

\section{Results}

\section{Sociodemographic Characteristics of Respondents}

A total of 1100 respondents, with a response rate of $98.3 \%$, were studied. Table 3 describes their sociodemographic characteristics. Of the 1100 participants sampled, 420 (38.2\%) [95\% Cl: 34.9 - 42.7] were drawn from Limbe, 320 (29.1\%) [95\% Cl: 25.4 - 33.9] from Tiko and 360 (32.7\%) [95\% Cl: 26.6 - 36.9] from Buea Health Districts following the principle of probability proportionate to size. Half (50.3\%) [95\% Cl: $47.3-53.3$ ] of the community members sampled were males. The mean age of the community members was $35.2(\mathrm{SD}=5.2)$ years and ranged from 21 - 78 years. Majority of the community members were either married or cohabiting 517(51.3\%) [95\% Cl: 48.2 54.4], were self-employed 391(35.7) [95\% Cl: $31.8-40.0$ ], had attained secondary school 572(53.7\%) [95\% Cl: 50.7 - 56.6] and were Christians 1033(97.4\%) [95\% Cl:96.2 - 98.1]. Majority 785(81.0\%) [95\% Cl:78.4 - 83.4] of the community members had an average monthly income less than 185USD. Natives (Bakwerians) constituted only 202(18.5\%) [95\% Cl: 16.3 - 20.4] of the sample. The mean number of people residing in a household was $5.67(\mathrm{SD}=1.2)$ and most $489(44.7 \%)$ [95\% Cl:41.8-47.7] of the households had between 4 - 6 members. Majority 786(76.0\%) [95\% Cl:73.3 - 78.5] of the community members resided in rural areas.

\section{Table 3: Sociodemographic characteristics of participants}




\begin{tabular}{|c|c|c|}
\hline Variables & $\begin{array}{l}\text { Frequency } \\
\text { No (\%) }\end{array}$ & $95 \% \mathrm{Cl}$ \\
\hline \multicolumn{3}{|l|}{ Age (years) } \\
\hline $21-30$ & $286(26.2)$ & $23.7-28.9$ \\
\hline $31-40$ & 363(33.2) & $30.5-36.1$ \\
\hline$\geq 41$ & $443(40.6)$ & $37.7-43.5$ \\
\hline Total & 1092(100) & \\
\hline \multicolumn{3}{|l|}{ Gender } \\
\hline Male & $538(50.3)$ & $47.3-53.3$ \\
\hline Female & $532(49.7)$ & $46.7-52.7$ \\
\hline Total & 1070(100) & \\
\hline \multicolumn{3}{|l|}{ Marital status } \\
\hline Single & $438(43.4)$ & $40.4-46.6$ \\
\hline Married & $517(51.3)$ & $48.2-54.4$ \\
\hline Widow/Divorced & $53(5.3)$ & $4.0-6.8$ \\
\hline Total & 1008(100) & \\
\hline \multicolumn{3}{|l|}{ Occupation } \\
\hline Civil servant & 151(13.8) & $11.9-15.9$ \\
\hline Private sector employee & $32(2.9)$ & $2.1-4.1$ \\
\hline Self-employed & 391(35.7) & $31.8-40.0$ \\
\hline Student & 392(35.8) & $33.0-38.9$ \\
\hline Unemployed & $129(11.9)$ & $8.7-15.3$ \\
\hline Total & 1095(100) & \\
\hline \multicolumn{3}{|l|}{ Religion } \\
\hline Christian & 1033(97.4) & $96.2-98.1$ \\
\hline Muslim & $17(1.6)$ & $1.0-2.5$ \\
\hline Traditionalist & $11(1.0)$ & $0.6-1.8$ \\
\hline Total & 1061(100) & \\
\hline \multicolumn{3}{|l|}{ Education } \\
\hline No formal education & $35(3.2)$ & $2.4-4.5$ \\
\hline Primary & $280(26.3)$ & $23.7-28.9$ \\
\hline Secondary & $572(53.7)$ & $50.7-56.6$ \\
\hline Tertiary & 179(16.8) & $14.7-19.5$ \\
\hline Total & 1066(100) & \\
\hline \multicolumn{3}{|l|}{ Ethnicity } \\
\hline Native (Bakwerian) & 202(18.5) & $16.3-20.4$ \\
\hline Others & 893(81.5) & $79.2-83.5$ \\
\hline Total & 1095(100) & \\
\hline
\end{tabular}

Page 6/18 


\begin{tabular}{|lll|}
\hline Monthly income (USD) & & \\
\hline$<185$ & $785(81.0)$ & $78.4-83.4$ \\
\hline $185-370$ & $160(16.5)$ & $14.3-18.9$ \\
\hline$>370$ & $24(2.5)$ & $1.7-3.7$ \\
\hline Total & $969(100)$ & \\
\hline Household size(persons) & & \\
\hline $1-3$ & $348(31.9)$ & $29.1-34.7$ \\
\hline $4-6$ & $489(44.7)$ & $41.8-47.7$ \\
\hline$>6$ & $256(23.4)$ & $21.0-26.0$ \\
\hline Total & $1093(100)$ & \\
\hline Health district & & \\
\hline Buea & $360(32.7)$ & $26.6-36.9$ \\
\hline Tiko & $320(29.1)$ & $25.4-33.9$ \\
\hline Limbe & $420(38.2)$ & $34.9-42.7$ \\
\hline Total & $1100(100)$ & \\
\hline Environmental setting & & \\
\hline Rural & $786(76.0)$ & $73.3-78.5$ \\
\hline Urban & $99(9.6)$ & $7.9-11.4$ \\
\hline Semi-urban & $149(14.4)$ & $12.4-16.7$ \\
\hline Total & $1034(100)$ & \\
\hline
\end{tabular}

USD = United states Dollars

\section{Prevalence of TCAM utilization by community members in the Fako Division}

Of the 1100 community respondents sampled, 754 (68.5\%) [95\% Cl: $66.3-71.5]$ had used TCAM in the last 12 months, $95 \% \mathrm{Cl}$ : $66.3-$ 71.5. A majority of the participants from the Tiko Health District 415(72.2\%) [95\% Cl: $70.6-73.6]$ use TCAM therapy followed by Buea Health District 328(69.9\%) [95\% Cl: 72.6 - 72.6] and lastly by the Limbe Health District 284 (63.9\%) [95\% Cl: 62.0 - 66.7] (Figure 1)

\section{Category of persons using TCAM products and practitioners}

Regarding the proportion of community members using TCAM, $42.8 \%$ [ $95 \% \mathrm{Cl}: 39.9$ - 45.7] were adults, $23.8 \%$ [95\% Cl: 21.3 - 26.3] were elders, $18.6 \%$ [95\% Cl: 16.6 - 20.9] were pregnant women and 14.8\% [95\% Cl: 12.7 - 16.9] were children (Figure 2).

Of the 754 community members using TCAM, 463 (61.4\%) [95\% Cl: 58.5 - 64.3] used TCAM products (self-care and over-the-counter use), 141 (18.7\%) [95\% Cl: 16.4 - 21.0] visited a TCAM practitioner and 150 (20.0\%) [95\% Cl: 17.6 - 22.4] used both TCAM products and practitioner services in the past 12 months (Figure 3).

Based on the type of TCAM practitioner visited by the community members, $79.7 \%$ [95\% Cl: $77.3-82.1$ ] visited herbalists, $14.9 \%$ [ $95 \% \mathrm{Cl}$ : 12.8 - 17.0] visited traditional bone setters, $3.4 \%$ [95\% Cl: $2.3-4.5]$ visited diviners, $1.3 \%$ [95\% Cl: $0.6-1.9]$ visited traditional birth attendants and $0.7 \%$ [95\% Cl: $0.2-1.2]$ visited massage therapists (Figure 4).

Based on the type of diseases for which community members visited TCAM practitioners, $44.6 \%$ [95\% Cl: $41.7-47.5$ ] consulted because of acute diseases (disease < 3months), 43.4\% [95\% Cl: 40.5 - 46.3] visited traditional healers for chronic diseases (disease > 3months) and $12.0 \%$ [95\% Cl: 10.1 - 13.9] for health promotion and disease prevention (Figure 5). 
The proportion of community members reported they used herbal medicine to prevent COVID-19 was $24.4 \%$ (95\%Cl: $20.6-29.1)$. Of the 91 community respondents who used TCAM therapy, 22 (24.6\%) [95\%Cl:22.1-27.1] used garlics, 20 (22.5\%) [95\%Cl:20.0-24.9] used warm water, 18 (20.1\%) [95\%Cl:17.7 -22.5] used fever grass tea and 14 (15.4\%) [95\%Cl:17.5 -17.5] used lemon tea (Figure 6).

Boiling (Table 4) was the most common methods of preparing herbal medicine for the treatment of various ailments. Most of the herbal medicines required a mixture of many plants (concoction). The treatment duration ranged from 1 day to 7 days and the dosage was in glasses or cups (ranging from 1 glass to 3 glasses daily). The plants used were mostly from the gardens, herbalist, market, and environment or forest.

Table 4: Plants used by community members based on the diseases treated as derived from this study

\begin{tabular}{|c|c|c|c|c|c|c|c|c|c|}
\hline $\begin{array}{l}\text { Disease/Ailments } \\
\text { treated }\end{array}$ & Plant species use & Common name & $\begin{array}{l}\text { Part } \\
\text { of the } \\
\text { plant } \\
\text { use } \\
\end{array}$ & $\begin{array}{l}\text { Dosage } \\
\text { form } \\
\text { used }\end{array}$ & $\begin{array}{l}\text { Route of } \\
\text { administration }\end{array}$ & $\begin{array}{l}\text { Method of } \\
\text { preparation }\end{array}$ & $\begin{array}{l}\text { Duration } \\
\text { of } \\
\text { treatment }\end{array}$ & Frequency & $\begin{array}{l}\text { Source of } \\
\text { plants }\end{array}$ \\
\hline \multirow[t]{5}{*}{$\begin{array}{l}\text { COVID-19, lung } \\
\text { infections/Cough, }\end{array}$} & Azadirachta indica & $\begin{array}{l}\begin{array}{l}\text { Dogoyaro } \\
\text { (Neem) }\end{array} \\
\end{array}$ & leaves & Fluid & Mouth & $\begin{array}{l}\text { Boiling- } \\
\text { water }\end{array}$ & 3days & Twice daily & Herbalist \\
\hline & Allium sativum & garlic & roots & solid & Mouth & Mouth & 3days & Twice daily & Market \\
\hline & Zingiber officinale & Ginger & Roots & Solid & Mouth & Chewing & 2 days & Once daily & Environment \\
\hline & Artemisia annua & Quinine stick & $\begin{array}{l}\text { Entire } \\
\text { plant }\end{array}$ & Fever & Boiling-others & Mouth & 3days & Twice daily & Garden \\
\hline & Carica papaya & Pawpaw & Leaf & Fluid & Mouth & $\begin{array}{l}\begin{array}{l}\text { Boiling- } \\
\text { others }\end{array} \\
\end{array}$ & 7 days & Twice daily & Environment \\
\hline \multirow{6}{*}{$\begin{array}{l}\text { Stomach/GIT } \\
\text { disorders }\end{array}$} & Barbados aloe & Aloe vera & Leaf & Fluid & Mouth & Alone & 1day & Twice daily & Garden \\
\hline & Calpurnia aurea & - & Leaf & Fluid & Mouth & Alone & 3days & Twice daily & Forest \\
\hline & Garcinia kola & Bitter cola & Seed & Solid & Mouth & Alone & 1day & Once daily & Market \\
\hline & Senna alata & Eczema leaves & Leaf & Fluid & Mouth & $\begin{array}{l}\text { Boil + } \\
\text { moringa } \\
\text { (seed) }\end{array}$ & 2weks & $\begin{array}{l}\text { Thrice } \\
\text { daily }\end{array}$ & Garden \\
\hline & Spilantes filicaulis & Eye for fowl & Leaf & Solid & Mouth & Chewing & 3 days & Once daily & Garden \\
\hline & $\begin{array}{l}\text { Ocimum basilicum } \\
L\end{array}$ & Masepo(Bakweri) & Leaf & Solid & Mouth & Chewing & 2 days & Once daily & Environment \\
\hline \multirow[t]{7}{*}{$\begin{array}{l}\text { Fever (Febrile } \\
\text { illnesses) }\end{array}$} & Bidens Pilosa & Black jack & Leaf & Fluid & Mouth & $\begin{array}{l}\text { Boiling- } \\
\text { water }\end{array}$ & 3 days & $\begin{array}{l}\text { Twice } \\
\text { daily }\end{array}$ & Environment \\
\hline & Moringa oleifera & Moringa & Leaf & Fluid & Mouth & $\begin{array}{l}\text { Boiling- } \\
\text { water }\end{array}$ & 3 days & Twice daily & Herbalist \\
\hline & Cinchona officinalis & Quinine stick & Bark & Fluid & Mouth & $\begin{array}{l}\begin{array}{l}\text { Boiling- } \\
\text { others }\end{array} \\
\end{array}$ & 4 days & Twice daily & Market \\
\hline & $\begin{array}{l}\text { Cymbopogon } \\
\text { citratus }\end{array}$ & Fever grass & Leaf & Fluid & Mouth & $\begin{array}{l}\text { Boiling- } \\
\text { others }\end{array}$ & 3 days & Twice daily & Garden \\
\hline & Psidium guajava & Guava & Leaf & Solid & Mouth & Chewing & 1 day & $\begin{array}{l}\text { Thrice } \\
\text { daily }\end{array}$ & Environment \\
\hline & $\begin{array}{l}\text { Helianthus annuus } \\
L\end{array}$ & Sun flower & Leaf & Fluid & Mouth & $\begin{array}{l}\text { Boiling- } \\
\text { others }\end{array}$ & 7 days & Twice daily & Environment \\
\hline & Carica papaya & Pawpaw & Leaf & Fluid & Mouth & $\begin{array}{l}\text { Boiling- } \\
\text { others }\end{array}$ & 7 days & Twice daily & Environment \\
\hline
\end{tabular}

\section{Use of herbal medicines by traditional healers to treat COVID-19}

The most common signs and symptoms of COVID-19 reported by traditional healers was cough, catarrh, fever and joint pain. Boiling(concoction) was the most common methods of preparing herbal medicine for their patients. Parts of plants used ranged from leaves, roots, seeds to whole plants (Table 5).

Table 5: Common plants used by traditional healers for the treatment of COVID-19 


\begin{tabular}{|c|c|c|c|c|}
\hline Plant sp & $\begin{array}{l}\text { Common } \\
\text { name }\end{array}$ & Part of plant used & Symptoms of patient & Mode of preparation \\
\hline Allium cepa & Onion & dried leaves & Cough, Catarrh & Boiling \\
\hline Aloe barbadensis & Aloe vera & leaves & Cough, Fever & Boiling \\
\hline Anthocleista djalonensis & unbrella leaf & Bark & Cough, Fever & Boiling-others \\
\hline Artemisia annua & Quinine stick & Entire plant & Fever & Boiling-others \\
\hline Azadirachta indica & & $\begin{array}{l}\text { leaves, stem and } \\
\text { bark }\end{array}$ & Cough, Fever, joint pain & Boiling-others \\
\hline Cola nitida & red cola & leaves, seeds & $\begin{array}{l}\text { Headache, Fever, } \\
\text { Myalgia }\end{array}$ & $\begin{array}{l}\text { Burn macerate+palm } \\
\text { oil }\end{array}$ \\
\hline Curcuma longa & Tumeric & Roots & Cough, Fever, body weakness & Boiling \\
\hline Cymbopogon citratus & Fever grass & leaves & Joint pain, fatigue, headach & Boiling \\
\hline Eucalyptus globulus & Eucalyptus & leaves & Catarrh/Runny nose & Boiling \\
\hline Eugenia caryophyllata & Clove & Seeds & Cough, Catarrh, headach & Boiling \\
\hline Psidium guajava & Guava leaves & leaves & $\begin{array}{l}\text { Cough, fever, headach, sore } \\
\text { throat }\end{array}$ & Boiling \\
\hline Jatropha curcas & Big nuts plant & $\begin{array}{l}\text { leaves and } \\
\text { fruit(nuts) }\end{array}$ & sore throat, cough & Boiling \\
\hline Mangifera indica & Mango & bark, leaves & Cough, Fever, Catarrh & Boiling \\
\hline Melissa of cinalis & Lemon balm & leaves & Catarrh, Cough, Fever & Boiling \\
\hline Moringa Oleifera & Moringa & leaves and seeds & Cough, Catarrh & Boiling \\
\hline Panax ginseng & Ginseng & Roots & Cough, Fever & Boiling \\
\hline Paw paw leaves & & leaves & cough, fever & Boiling-others \\
\hline $\begin{array}{l}\text { Pennisetumpurpureum } \\
\text { Shum }\end{array}$ & King grass & Entire plant & Joint pain, fatigue & Boiling-others \\
\hline Pimpinella anisum & Aniseed & Fruit & Cough & Boiling \\
\hline Salvia officinalis & Sage & leaves & Sore throat, cough & Boiling \\
\hline Senna alata & ezema leaf & leaves & cough fever, joint pain & Boiling-others \\
\hline Thymus vulgaris & Garden thyme & leaves & Cough, Catarrh & Boiling \\
\hline
\end{tabular}

\section{Predictors of TCAM use in the communities.}

Multiple logistic regression model showed that age more than 41 years old had 2.23 (95\% Cl: 1.09-4.91) times the odds of using TCAM than people between 21-30years. A farmer had 3.05 (95\% Cl: 1.99-5.34) times the odds of using TCAM than people from other occupations. Haven attained tertiary education 0.33 (95\% Cl: $0.16-0.88$ ) were less likely associated with TCAM use compared to those with no formal education. Community members having between 100,000 - 200,000FCFA as monthly income had 2.06 (95\% Cl: 1.33-4.55) times the odds of using TCAM than those with income levels below 100,000Fcfa. Participants who resided in a rural setting had 2.30 (95\% Cl: 1.04-3.98) times the odds of using TCAM than those residing in an urban setting, being knowledgeable on TCAM had 3.10 (95\% Cl: 1.54-6.45) times the odds of using TCAM than not being knowledgeable on TCAM and having a positive attitude towards TCAM had 3.47 (95\% Cl: 1.94-6.45) times the odds of using TCAM than those with a negative attitude towards TCAM. (Table 6).

\section{Table 6: Determinants of TCAM use in the communities}




\begin{tabular}{|c|c|c|c|c|c|c|}
\hline \multirow[t]{2}{*}{ Variable } & \multicolumn{3}{|c|}{ Use of TCAM } & & \multirow{3}{*}{ p-value } \\
\hline & \multirow{2}{*}{$\begin{array}{l}\text { TCAM use } \\
\mathrm{N}(\%)\end{array}$} & \multirow{2}{*}{$\begin{array}{l}\text { TCAM non-use } \\
\mathrm{N}(\%)\end{array}$} & \multirow[t]{2}{*}{ AOR } & & & \\
\hline & & & & \multicolumn{2}{|r|}{ (95\%Cl) } & \\
\hline \multicolumn{7}{|l|}{ Age (years) } \\
\hline $21-30$ & $177(62.1)$ & $108(37.9)$ & \multicolumn{4}{|l|}{1} \\
\hline $31-40$ & $239(65.8)$ & $124(34.2)$ & 1.11 & \multicolumn{2}{|c|}{$0.81-3.68$} & 0.138 \\
\hline$\geq 41$ & $336(75.8)$ & $107(24.2)$ & 2.23 & \multicolumn{2}{|c|}{$1.09-4.91$} & $0.018^{*}$ \\
\hline Total & 752 & 339 & & & & \\
\hline \multicolumn{7}{|l|}{ Gender } \\
\hline Male & $385(71.6)$ & $153(28.4)$ & 1 & & & \\
\hline Female & $354(66.7)$ & $177(33.3)$ & 0.71 & \multicolumn{2}{|r|}{$0.45-2.15$} & 0.113 \\
\hline Total & 739 & \multicolumn{2}{|l|}{330} & & & \\
\hline \multicolumn{7}{|l|}{ Marital status } \\
\hline Single & $287(65.7)$ & $150(34.3)$ & 1 & & & \\
\hline Married/Cohabiting & $364(70.4)$ & $153(29.6)$ & 1.09 & \multicolumn{2}{|r|}{$0.84-1.42$} & 0.512 \\
\hline Widow/Divorced & $43(81.1)$ & $10(18.9)$ & 1.13 & \multicolumn{2}{|r|}{$0.15-1.23$} & 0.124 \\
\hline Total & 694 & \multicolumn{2}{|l|}{313} & & & \\
\hline \multicolumn{7}{|l|}{ Employment status } \\
\hline Employed & $395(68.8)$ & $179(31.2)$ & 1 & & & \\
\hline Unemployed & $359(69.0)$ & $161(31.0)$ & 1.01 & & $0.80-3.09$ & 0.534 \\
\hline Total & 754 & 340 & & & & \\
\hline Occupation & & & & & & \\
\hline Others & $700(66.9)$ & $346(33.1)$ & 1 & & & \\
\hline Farming & $54(87.1)$ & $8(12.9)$ & 3.04 & & $1.99-5.34$ & $0.002^{\star}$ \\
\hline Total & 754 & 354 & & & & \\
\hline Education & & & & & & \\
\hline None & $26(74.3)$ & $9(25.7)$ & 1 & & & \\
\hline Primary & $197(70.4)$ & $83(29.6)$ & 0.8 & 0. & $74-4.23$ & \\
\hline Secondary & $405(70.9)$ & $166(29.1)$ & 0.9 & 0. & $26-4.15$ & \\
\hline Tertiary & $105(58.7)$ & $74(41.3)$ & 0.3 & 0 . & $16-0.88$ & $01 *$ \\
\hline Total & 733 & 332 & & & & \\
\hline Household size(pers & & & & & & \\
\hline $1-3$ & $228(65.5)$ & $120(34.5)$ & & & & \\
\hline $4-6$ & $336(68.7)$ & 153 (31.3) & & 81 & $0.65-1.15$ & 0.243 \\
\hline$>6$ & 188 (73.7) & $67(26.3)$ & & 01 & $0.87-3.21$ & 0.255 \\
\hline Total & 752 & 340 & & & & \\
\hline Religion & & & & & & \\
\hline Christians & 717 (69.5) & 315 (30.5) & & & & \\
\hline
\end{tabular}




\begin{tabular}{|c|c|c|c|c|c|}
\hline Muslim & $9(52.9)$ & $8(47.1)$ & 0.66 & $0.45-1.07$ & 0.163 \\
\hline Traditionalist & $5(45.5)$ & $6(54.5)$ & 0.78 & $0.54-2.35$ & 0.235 \\
\hline Total & 731 & 329 & & & \\
\hline \multicolumn{6}{|c|}{ Monthly income(USD) } \\
\hline$<185$ & $527(67.2)$ & $257(32.8)$ & 1 & & \\
\hline $185-370$ & $127(79.4)$ & $33(20.6)$ & 2.06 & $1.33-4.55$ & 0.004 \\
\hline$>370$ & $10(41.7)$ & $14(58.3)$ & 0.41 & $0.23-0.98$ & $0.012^{*}$ \\
\hline Total & 664 & 304 & & & \\
\hline \multicolumn{6}{|l|}{ Ethnicity } \\
\hline Native (Bakwerian) & $140(69.3)$ & $62(30.7)$ & 1 & & \\
\hline Others & $614(68.8)$ & 278 (31.2) & 0.87 & $0.34-2.45$ & 0.97 \\
\hline Total & 754 & 340 & & & \\
\hline \multicolumn{6}{|c|}{ Environmental setting } \\
\hline Urban & $558(71.1)$ & $227(28.9)$ & 1 & & \\
\hline Semi-urban & $69(69.7)$ & $30(30.3)$ & 1.12 & $0.89-2.35$ & 0.541 \\
\hline Rural & $86(57.7)$ & $63(42.3)$ & 2.30 & $1.04-3.98$ & $0.022 *$ \\
\hline Total & 713 & 320 & & & \\
\hline \multicolumn{6}{|l|}{ Knowledge on TCAM } \\
\hline Incorrect & $212(64.4)$ & $117(35.6)$ & 1 & & \\
\hline Correct & $542(70.3)$ & $229(29.7)$ & 3.10 & $1.54-6.45$ & 0.010 * \\
\hline Total & 754 & 346 & & & \\
\hline \multicolumn{6}{|c|}{ Attitude towards TCAM } \\
\hline Negative & $404(66.0)$ & $208(34.0)$ & 1 & & \\
\hline Positive & $350(71.7)$ & $138(28.3)$ & 3.47 & $1.94-6.45$ & $0.010 *$ \\
\hline Total & 754 & 346 & & & \\
\hline
\end{tabular}

USD = United States Dollar

\section{Discussion}

\section{Prevalence and Pattern of TCAM use in the study communities}

This study revealed some interesting findings, some of which tally with literature and some that do not tally with prevailing trends of traditional, complementary and alternative medicine (TCAM) use. This study shows the prevalence of TCAM therapy use to be $68.5 \%$ which is lower than the $74.22 \%$ reported in Ethiopia, $77.5 \%$ reported in Nigeria [14] and also less than the $80 \%$ reported by the WHO. It was however greater than the prevalence of $58.2 \%$ obtained in a systematic survey for Sub Saharan Africa [6], and greater than the $60.0 \%$ TCAM use among respondents in Zimbabwe [15] and the 592 (55.5\%) reported in Malaysia [16]. Differences in prevalence may be due to differences in study sites and in the sample sizes, as most studies in the systematic survey for SSA had sample sizes less than 500 . The prevalence of TCAM use in Cameroon was much higher than that reported elsewhere in the industrialized world, ranging between about $29 \%$ in the USA [17] to about $49 \%$ in Australia [18].

Based on the use of TCAM therapy by health District, Tiko (72.2\%) and Buea (69.9\%) Health districts recorded the highest users of TCAM therapy as compared to Limbe(63.9\%) and this difference was statistically significant $(X=5.632 p=0.056)$. This might be due to 
differences in location with the availability and accessibility to natural plants, coupled with mount Fako in Buea which is rich in diverse plant flora compared to Limbe. The majority of TCAM users were adults (42.8\%), Elders (23.8\%) and Pregnant women (18.6\%) while children (14.8\%) were the least TCAM users. These population category was slightly different from that in Ethiopia where by complementary and alternative medicine was most commonly given to the elderly (32.62\%), adults (24.11\%), followed by children (17.02\%) and pregnant women (2.13\%) [19]. The prevalence of herbal medicine use among pregnant women was $18.6 \%$ which was less than that obtained in Nigeria 36.8\% [20] but greater than the 2.13\% [21] obtained in Ethiopia. This can be due to the differences in the spatial distribution of the sample population. TCAM is highly use among the young adults despite their level of education and access to orthodox health services, which show that the knowledge and usage of TCAM is evergreen regardless of the ancient age of TCAM. Among the users of TCAM therapy, $61.4 \%$ use TCAM products while $18.7 \%$ visited TCAM practitioners only, while $20.0 \%$ use both. Studies conducted in Africa shows the prevalence of TCAM product use in the general population [22-24] ranging from 4.6\% (urban settlement in Ethiopia) [25] to 94\% (semi urban settlements in Nigeria and Ethiopia) [26-27], with an estimated average of 58.2\%. Some studies [28-32] reported on TCAM practitioner utilization (1.2\%-67\% (mean, 28.8\%). A lower prevalence (1.2\%-44.1\% (average, 12.6\%) of TCAM practitioner services use was observed in studies with large sample sizes [32-35] compared with other studies [35, 36-37] with smaller samples (37.5\%-67\% (mean, 53.0\%). This was in line with our study with a large sample size of 1100 participants in the general population recording an $18.7 \%$ of TCAM practitioner use compared with smaller size sample sizes. This prevalence of TCAM practitioner use may be higher in disease specific subpopulations compared to the general population. Peltzer. et al (2016) [5] obtained prevalence of TCAM provider use, in Australia 34.7\%, in Europe ranging from under 10\% in Bulgaria, Poland and Slovenia to $35.4 \%$ in France, in Asia from $16.7 \%$ in Russia to over $50 \%$ in China mainland, the Philippines and Republic of Korea, and over $20 \%$ in the USA, Chile and South Africa. The majority of the community members mostly use the services of herbalists, bone setters, followed by diviners. Similar findings were obtained in the Northwest Region of Cameroon where mostly the services of herbalists, traditional bone setters, diviners and traditional birth attendants where sorted for by the general population [38]. Our findings were similar to that in Tanzania where diviners, herbalists, traditional birth attendants, and bone setters were mostly visited by the general population [39]. Close to half of the population use TCAM for acute conditions (44.6\%) or for chronic (43.6\%) conditions while a small proportion use TCAM to maintain wellbeing. Our findings were not congruent with that obtained by Peltzer. K et al (2019) [40], in which 53.7\% of the participants used TCAM for treating a chronic or long-term health condition, $40.0 \%$ used TCAM in order to improve well-being and $6.3 \%$ for treating an acute illness. According to Bannerman RH et al., [41] complementary and alternative medicine is used by the people for the management of chronic conditions that are costly to society, such as chronic pain and arthritis, and more life-threatening diseases such as heart disease and cancer, which gives strength to the TCAM than orthodox, thus a need for integration to overcome the weaknesses of each.

\section{Community use of TCAM to prevent COVID-19}

Allium sativium (garlic), Azadirachta indica, Zingiber officinale, Artemisia annua, Carica papaya were mostly used in combination with other plants to prevent COVID-19 in the communities. Our findings were similar to that obtained in previous studies i.e in Madagascar a combination of artemisia, neem leaves, paw leaves, garlic, ginger, lime and oranges has been adopted as a notable anti-COVID agent [42]. These herbs are boiled together for $30 \mathrm{~min}$ and steam-inhaled. Expectedly the steam inhalation clears the lungs of the virus [42]. Our findings were also in line with that obtained in Nigeria in which phenolic compounds and antioxidant properties of these herbal remedies are known to contribute to their therapeutic effects [43]. Consumption of these Nigerian herbal remedies increase the anti-oxidant molecules and enzymes in the body and protect the cells and its membrane from being damaged by the toxic substances [43,44]. Phenolic moieties boost the body's immunity and defense against the threatening virus [45]. Scientists from the academia and Research Institutes have also supported the use of these herbal remedies as anti-COVID agents.

\section{Herbal preparations used by traditional healers for COVID-19}

This study brings out a number of herbal remedies used by traditional healers for COVID-19 which was congruent with that obtained by Fongnzossie et al (2021) [46] who reported the use of herbal remedies including Azadirachta indica, Zingiber officinale, Artemisia annua, Carica papaya for the treatment of COVID-19 with symptoms such as sore throat, cough, catarrh, fever and jointpains. Azadiracta indica (neem) is one of the most used plants in the treatment of malaria in many parts of Africa and Asia where malaria is endemic. Roger, et al.(2020) provides proof that the neem plant can provide anti-viral effects for COVID-19[47]. Similar plants species were reported as potential agents for COVID-19 in Ethiopia [48]. These included, Lepidium sativum [48], Azadirachta indica [49], Osyris quadripartite [50] and Allium sativum [51]. Citrus aurantium L., Citrus limon (L.) Burm. f., Capsicum annuum L., Eucalyptus globulus, Osyris quadripartite, Amaranthus hybridus Linn were also cited as effective against COVID-19 [51].

\section{Determinants of TCAM use in the General population}


Age above 41years, farming as occupation, having attained tertiary education, having a monthly income above 185USD, residing in a rural setting, being knowledgeable on TCAM and having a positive attitude towards TCAM were strongly associated with the use of TCAM. However, age group was different compared with other study as ages between 18-28 and 29-38 years were associated with the use of TCAM in Ethiopia [52]. Similar findings were obtained in the Bui-Division of Cameroon where most patients reported visiting the traditional healers because of the low cost (69\%) and low level of income [53].

Our findings were also congruent with Karl et al [40] in which middle age, being a female, lower educational status, not having a religious affiliation, larger household size, not having a health insurance, could not pay for medical treatment when needed it were associated with TCAM provider use. Also the authors showed that having a chronic condition or disability, and having positive attitudes towards TCAM (TCAM being better than mainstream medicine and TCAM does not promise more than it can deliver) significantly increased the odds of TCAM provider use. However, our findings were different from that obtained from Cambodia whereby being a female $(A O R=1.42,95 \% \mathrm{Cl}$ $=1.12-2.67)$, haven completed less schooling $(\mathrm{AOR}=0.66,95 \% \mathrm{Cl}=0.45-0.96)$, unemployed or homemakers $(\mathrm{AOR}=0.23,95 \% \mathrm{Cl}=$ 0.13-0.52) and have a gastrointestinal illness ( $A O R=0.49,95 \% \mathrm{Cl}=0.39-0.62)$ were associated with TCAM use [54]. Findings were similar to that obtained in Ethiopia whereby age $(P=0.02)$, especially age group between 18 and 28 and 29 and $38((P=0.02$ and 0.004 , resp.), educational status was also significantly associated with TCAM use $(P=0.00)$. Moreover, occupation $(P=0.00)$ and effectiveness of TCAM (0.002) were found to be associated with TCAM use [52]. Our findings were however different from study in the general population in Ghana which shows that TCAM use was predicted by having low-income levels [odds ratio (OR) 2.883, confidence interval (CI) 1.1427.277], being a trader (OR 2.321, $\mathrm{Cl} 1.037-5.194)$, perceiving TCAM as effective (OR 4.430, Cl 1.645-11.934) and safe (OR 2.730, Cl 0.986-4.321), good affective behavior of traditional medical practitioner (TMP) (OR 2.943, Cl 0.875-9.896) and having chronic ill-health (OR 3.821, Cl 1.213-11.311) were associated with TCAM use [55]. Differences in the predictors of TCAM use may be due to difference in study population.

Prevalence of TCAM use was measured within a standard pattern of TCAM use within the past 12 months. As a limitation to this study there is the possibility of recall bias and thus an under or over reporting of prevalence for TCAM use. Thus to overcome this, further questions were asked and a large sample size to overcome such limitations.

\section{Conclusion}

Our study found that TCAM are broadly used among community members in the Fako Division of Cameroon. More than half of the population having used TCAM in the last 12 months. The most commonly used TCAM was herbals. Allium sativum, Azadirachta indica, Zingiber officinale, Artemisia annua were most commonly used TCAM for COVID-19. Occupation, level of education, monthly income, environmental setting, good knowledge and positive attitudes were associated with TCAM use. Since the importance of TCAM is well accepted and used by the population and officially supported by WHO, integration of TCAM into the primary health care system should be further encouraged through policy, efficacy and safety and supported by more specific research in Cameroon. Based on our findings it can be concluded that medicinal plants can be promising resources for the management of COVID-19 based on population utilization and by traditional healers on their patients for COVID-19 which can further be tested for efficacy and toxicity as potential COVID-19 regimens. Also, policies and healthcare interventions sensitive to TCAM should be tailored mindful of the wide use of TCAM products and traditional healers in the general population of Southwest Region of Cameroon.

\section{Abbreviations}

TCAM-Traditional, Complementary and Alternative Medicine

TM-Traditional Medicine

COVID-19- Coronavirus Disease 2019

THP- Traditional Health Practitioners

OHPs - Orthodox Health Practitioners

OM - Orthodox medicine

WHO-World Health Organization

UHC-Universal Health Coverage 


\section{Declarations}

\section{Ethics approval and consent to participate}

Ethical clearance was obtained from the Faculty of Health Sciences Institutional Review Board (FHSIRB) (Reference Number: 2018/95203) of the University of Buea. Following the review and approval of the research proposal by FHSIRB, an administrative authorization was obtained from the Regional Delegation of Public Health, South West Region. Each study participant agreed to participate voluntarily. Participants were allowed to discontinue the interview when they needed. All participants of the study declared their willingness to participate and approved by their written and verbal consents. Participant's information was kept confidential and codes were used on the questionnaire and interview guides.

\section{Consent for publication}

Not applicable

\section{Availability of data and materials}

The datasets used and/or analyzed during the current study are available from the corresponding author on reasonable request.

\section{Competing Interests}

This study was conducted independent of employment and the author declares no financial assistance was given nor any financial gain is promised as a result of publication. The authors declare that they have no competing interests.

\section{Funding}

Not applicable

\section{Authors' Contributions}

ACW participated in the conception, designing, conducted the data collection, analysis and drafting of manuscript. NNT participated in the drafting, read and corrected the manuscript; ANJC participated in designing, supervision, drafting of the manuscript and substantially revised the manuscript for academic content. NDS participated in the conception, oversaw the data collection process and analysis, supervised the work and substantially revised the manuscript for academic content. All authors read and approved the final copy of the manuscript.

\section{Acknowledgement}

The authors will like to express their sincere appreciation to the community respondents, the community leaders including some local chiefs that were visited as bottle necks and the Regional Delegation of Public Health for the South west Region who have contributed in making this study possible and successful.

\section{References}

1. World Health Organization, editor. WHO traditional medicine strategy 2014-2023. Geneva: World Health Organization; 2013.76 p. 2. World Health Organization Promoting the role of traditional medicine in health systems: a strategy for the African Region, 2000. 3. Yu LP \& Rachel C. Integrating Traditional and Complementary Medicine with National Healthcare Systems for Universal Health Coverage in Asia and the Western Pacific, Health Systems \& Reform, (2019) 5:1, 24-31 4. WHO I Traditional medicine. World Health Organization; Available at: http://www.who.int/traditional-complementary-integrative-medicine/about/en. Assessed on 18th Feb 2021. 5. Peltzer K, Pengpid S. Prevalence and determinants of traditional, complementary and alternative medicine provider use among adults from 32 countries. Chin J Integr Med 2018;24:584-90. 10.1007/s11655-016-2748-y. 6. James PB, Wardle J, Steel A, et al. Traditional, complementary and alternative medicine use in Sub-Saharan Africa: a systematic review BMJ Global Health 2018;3:e000895. 7. Bishop FL, Lewith GT. Who Uses CAM? A Narrative Review of Demographic Characteristics and Health Factors Associated with CAM Use. Evid-Based Complement Altern Med ECAM. 2010 Mar;7(1):11-28. 8. Oluyemi Joseph, Yinusa Muhammed, Abdullateef Raji, Adejoke Joseph. Utilization of Herbal Medicine Among Inhabitants of an Urban Centre in North-Central Nigeria. Algerian J Nat Prod. 2016 Dec 28;4(3):367-78. 9. Mavhunga C. 
Zimbabwe's government says herbal treatment OK for COVID-19 | voice of America - English. Voice of America. Available at: https://www.voanews.com/science-health/coronavirus-outbreak/zimbabwes-government-says-herbal-treatment-ok-covid-19 10. Huang J, Su D, Feng Y, Liu K, Song Y. Antiviral herbs--present and future. Infect Disord Drug Targets. 2014;14(1):61-73. 11. Panyod S, Ho C-T, Sheen L-Y. Dietary therapy and herbal medicine for COVID-19 prevention: A review and perspective. J Tradit Complement Med. 2020 May 30;10(4):420-7. 12. Egbe AE, Tabot PT, Fonge BA. Ethnobotany of Some Selected Tree Species in Southwest Cameroon. Ethnobot Res Appl. 2012 Jun 17;10:235. 13. Quandt SA, Verhoef MJ, Arcury TA, Lewith GT, Steinsbekk A, Kristoffersen AE, et al. Development of an international questionnaire to measure use of complementary and alternative medicine (I-CAM-Q). J Altern Complement Med. N Y N. 2009 Apr;15(4):331-9. 14. Chukwuma BD, Kevin CD, Kenechi AU, Chioma AD, Irene AM, et al. Combined Orthodox and Traditional Medicine Use among Households in Orlu, Imo State, Nigeria: Prevalence and Determinants. World Journal of Preventive Medicine. Vol. 4, No. 1, 2016, pp 5-11. 15. Walter C and Jerneja V. Prevalence, perceptions and factors influencing the use of traditional and complementary medicine (T\&CM) in Zimbabwe's adult population: The case of Bindura District. European Journal of Integrative Medicine, 2016 ISSN: 1876-3820, Vol: 8, Issue: 4, Page: 484-493. 16. Mohamad TA, Islahudin F, Jasamai M, Jamal JA. Preference, perception and predictors of herbal medicine use among Malay women in Malaysia. Patient Prefer Adherence. 2019;13:1829. 17. Eisenberg DM, Kessler KC, Foster C, Norlock FE, Calkins DR, Delbanco TL. Unconventional Medicine in the United States - Prevalence, Costs, and Patterns of Use. N. Engl. J. Med. 328 (1993) 246-252 18. World Health Organization 2002. Traditional medicine. Available at https://www.who.int/intellectualproperty/topics/traditional/en/. Assessed on the 16/02/2021. 19. Belachew N, Tadesse T, Gube AA. Knowledge, attitude, and practice of complementary and alternative medicine among residents of Wayu town, Western Ethiopia. J Evid Based Complement Altern Med. 2017;22(4):929-35. 20. Chukwuma BD, Kenechi AU, Nnebue CC, Ikechukwu IM, Kevin CD, et al. “Sociodemographic Determinants of Herbal Medicine Use in Pregnancy Among Nigerian Women Attending Clinics in a Tertiary Hospital in Imo State, South-East, Nigeria." American Journal of Medicine Studies, vol. 4, no. 1 (2016): 1-10. doi: 10.12691/ajms-4-1-1. 21. Okoronkwo I, Onyia-pat J-lovena, Okpala P, et al. . Patterns of complementary and alternative medicine use, perceived benefits, and adverse effects among adult users in Enugu Urban, Southeast Nigeria. Evid Based Complementary Altern Med 2014;2014:1-6. 10.1155/2014/239372. 22. Onyiapat JL, Okoronkwo IL, Ogbonnaya NP. Complementary and alternative medicine use among adults in Enugu, Nigeria. BMC Complement Altern Med 2011;11:19 10.1186/1472-6882-11-19. 23. Galabuzi C, Agea J, Fungo B. Traditional medicine as an alternative form of health care system: a pre liminary case study of Nangabo sub-county, central Uganda. Afr J Tradit Complement Altern Med 2010;7:11-16. 10.4314/ajtcam.v7i1.57224 24. Abodunrin OL, Omojasola T, Rojugbokan OO. Utilization of alternative medical services by people of a north central city of Nigeria. East Afr J Public Health 2011;8:82-7 25. Flatie T, Gedif T, Asres K, et al. . Ethnomedical survey of Berta ethnic group Assosa Zone, Benishangul-Gumuz regional state, mid-west Ethiopia. J Ethnobiol Ethnomed 2009;5:14 10.1186/17464269-5-14 26. Gari A, Yarlagadda R, Wolde-Mariam M. Knowledge, attitude, practice, and management of traditional medicine among people of Burka Jato Kebele, West Ethiopia. J Pharm Bioallied Sci 2015; 7:136 10.4103/0975-7406.148782 27. Sarki ZM . Sociodemographic factors and utilization of traditional medicine in Kazaure Town, Jigawa State, Nigeria. Int Journal of Emerging Knowledge 2015;3:9-20. 28. Nxumalo N, Alaba O, Harris B, et al. . Utilization of traditional healers in South Africa and costs to patients: findings from a national household survey. J Public Health Policy 2011;32 Suppl 1:S124-S136. 10.1057/jphp.2011.26 29. Oyebode 0, Kandala NB, Chilton PJ, et al. . Use of traditional medicine in middle-income countries: a WHO-SAGE study. Health Policy Plan 2016;31:984-91. 10.1093/heapol/czw022 30. Birhan W, Giday M, Teklehaymanot T. The contribution of traditional healers' clinics to public health care system in Addis Ababa, Ethiopia: a cross-sectional study. J Ethnobiol Ethnomed 2011;7:39 10.1186/1746-4269-7-39 31. Mathibela MK, Egan BA, Du Plessis HJ, et al. . Socio-cultural profile of Bapedi traditional healers as indigenous knowledge custodians and conservation partners in the Blouberg area, Limpopo Province, South Africa. J Ethnobiol Ethnomed 2015;11:49 10.1186/s13002-015-0025-3 32. Mbereko A, Mahlatini P. Understanding contributions of traditional healers to the prevention, care and support in the fight against HIV and AIDS Pandemic in Kariba, Zimbabwe. Int J Sociol Anthropol 2014;6:136. 33. De Jager GF, Prinsloo EAM, Joubert G. Use of traditional medicine versus use of the community-based primary health care clinic by the San community at Platfontein. South African Family Practice 2010;52:542-7. 10.1080/20786204.2010.10874045 34. Birhan W, Giday M, Teklehaymanot T. The contribution of traditional healers' clinics to public health care system in Addis Ababa, Ethiopia: a cross-sectional study. J Ethnobiol Ethnomed 2011;7:39 10.1186/1746-4269-7-39 35. Agbor, AM, Naidoo, S. 2011. Knowledge and practice of traditional healers in oral health in the Bui Division, Cameroon. J Ethnobiol Ethnomed. 7:6. 36. Stanifer JW, Patel UD, Karia F, et al. . The determinants of traditional medicine use in northern Tanzania: a mixed-methods study. PLoS One 2015;10:e0122638 10.1371/journal.pone.0122638 37. Bakshi SS, McMahon S, George A, et al. . The role of traditional treatment on health care seeking by caregivers for sick children in Sierra Leone: results of a baseline survey. Acta Trop 2013;127:46-52. 10.1016/j.actatropica.2013.03.010 38. Diaz T, George AS, Rao SR, et al. . Healthcare seeking for diarrhoea, malaria and pneumonia among children in four poor rural districts in Sierra Leone in the context of free health care: results of a crosssectional survey. BMC Public Health 2013;13:157 10.1186/1471-2458-13-157 39. Ranasinghe S, Ansumana R, Lamin JM, et al. . Herbs and herbal combinations used to treat suspected malaria in Bo, Sierra Leone. J Ethnopharmacol 2015;166:200-4.

10.1016/j.jep.2015.03.028 40. Peltzer K, Pengpid S. Prevalence and determinants of traditional, complementary and alternative medicine

Page 15/18 
provider use among adults from 32 countries. Chin J Integr Med 2018;24:584-90. 10.1007/s11655-016-2748-y. 41. Bannerman RH. The role of traditional medicine in primary health care: Traditional medicine and health care coverage. Geneva: WHO, 1983: 318-27. 42. Baker A. Unproven herbal cure for coronavirus is a hit in Africa. Available from: https://time.com/5840148/coronavirus-cure-covid-organicmadagascar/ . 43. Amadi CN, Offor SJ, Frazzoli C, Orisakwe O.E. Natural antidotes and management of metal toxicity. Environ. Sci. Pollut. Res. 2019:1-21. 44. Orisakwe OE., Amadi C.N., Frazzoli C., Dokubo A. Nigerian foods of probiotics relevance and chronic metal exposure: a systematic review. Environ. Sci. Pollut. Res. 2020:1-13. 45. Mondal S, Mirdha BR. The science behind sacredness of Tulsi (Ocimum sanctum Linn.) Indian J. Physiol. Pharmacol. 2009;53:291-306. 46. Fongnzossie F, Biwole E, Nyangono B, et al. A review of Cameroonian medicinal plants with potentials for the management of the COVID-19 pandemic. ADV TRADIT MED (ADTM) (2021). Available at: https://doi.org/10.1007/s13596-021-00567-6. 47. Can antimalarial, antiviral and anti-respiratory infections Cameroonian medicinal plants be used as one of the potential ways to cure COVID-19? Pharmacological and ethnomedicinal proof. Available at: https://www.researchgate.net/publication/343091749_Can_antimalarial_antiviral_and_antirespiratory_infections_Cameroonian_medicinal_plants_be_used_as_one_of_the_potential_ways_to_cure_COVID19_Pharmacological_and_ethnomedicinal_proof accessed Jun 23 2021. 48. Sharma AD and Kaur I. "Jensenone from eucalyptus essential oil as a potential inhibitor of COVID 19 Coronavirus infection," Research \& Reviews in Biotechnology \& Biosciences, vol. 7, no. 1, pp. 59-66, 2020. 49. Alzohairy MA, "Therapeutics role of Azadirachta indica (Neem) and their active constituents in diseases prevention and treatment," Evidence-Based Complementary and Alternative Medicine, vol. 2016, Article ID 7382506, 11 pages, 2016. 50. Shyaula S, "A review on genus Osyris: phytochemical constituents and traditional uses," Journal of Natural Pharmaceuticals, vol. 3, no. 2, p. 61, 2012. 51. Thuy BT, My TT, Hai NT et al., "Investigation into SARS-CoV-2 resistance of compounds in garlic essential oil," ACS Omega, vol. 5, no. 14, pp. 8312-8320, 2020. 52. Wassie SM, Aragie LL, Taye BW, et al. . Knowledge, attitude, and utilization of traditional medicine among the communities of merawi town, northwest ethiopia: A cross-sectional study 53. Agbor, AM, Naidoo, S. 2011. Knowledge and practice of traditional healers in oral health in the Bui Division, Cameroon. J Ethnobiol Ethnomed. 7:6. 54. Pearson H, Fleming T, Chhoun P, Tuot S, Brody C, Yi S. Prevalence of and factors associated with utilization of herbal medicines among outpatients in primary health centers in Cambodia. BMC Complement Altern Med. 2018 Apr 2;18(1):114. doi: 10.1186/s12906-018-2181-1. PMID: 29609580; PMCID: PMC5879807. 55. Gyasi RM, Mensah CM. \& Siaw LP. Predictors of Traditional Medicines Utilisation in the Ghanaian Health Care Practice: Interrogating the Ashanti Situation. J Community Health 40, 314-325 (2015). https://doi.org/10.1007/s10900-014-9937-4.

\section{Figures}

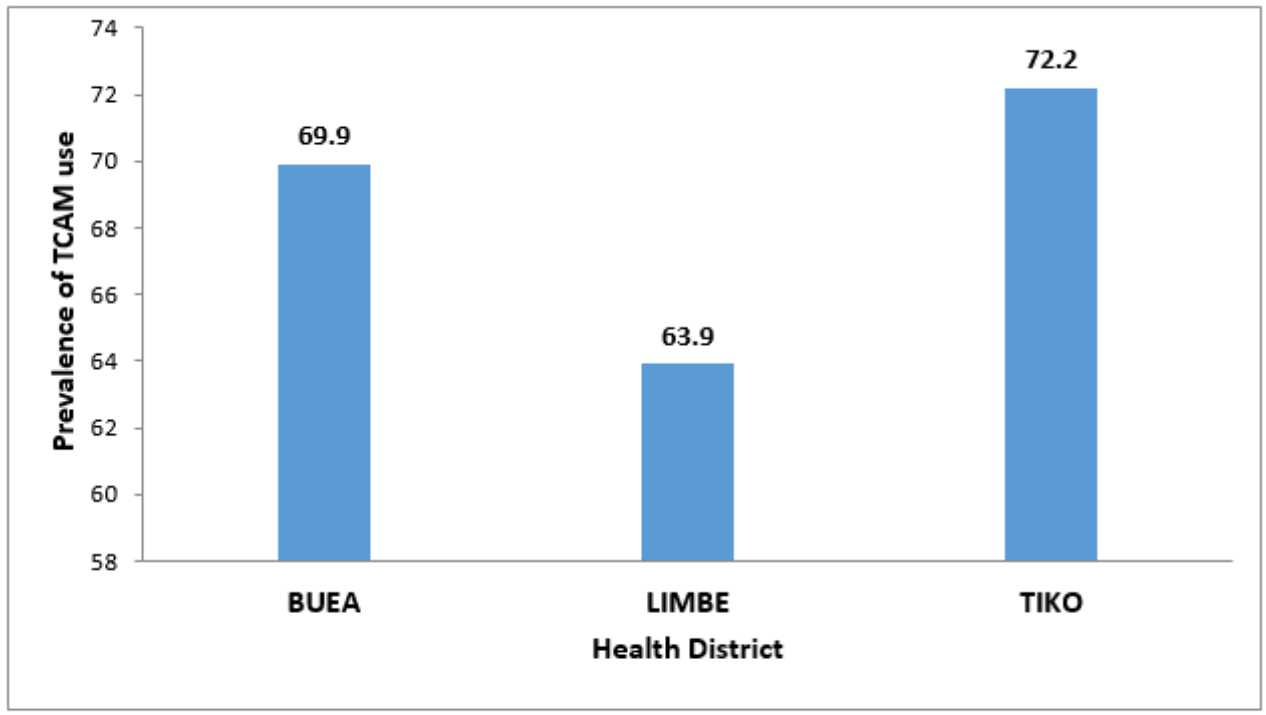

$X^{2}=5.632, \quad \mathrm{p}=0.056$

\section{Figure 1}

Prevalence of TCAM use per Health District 


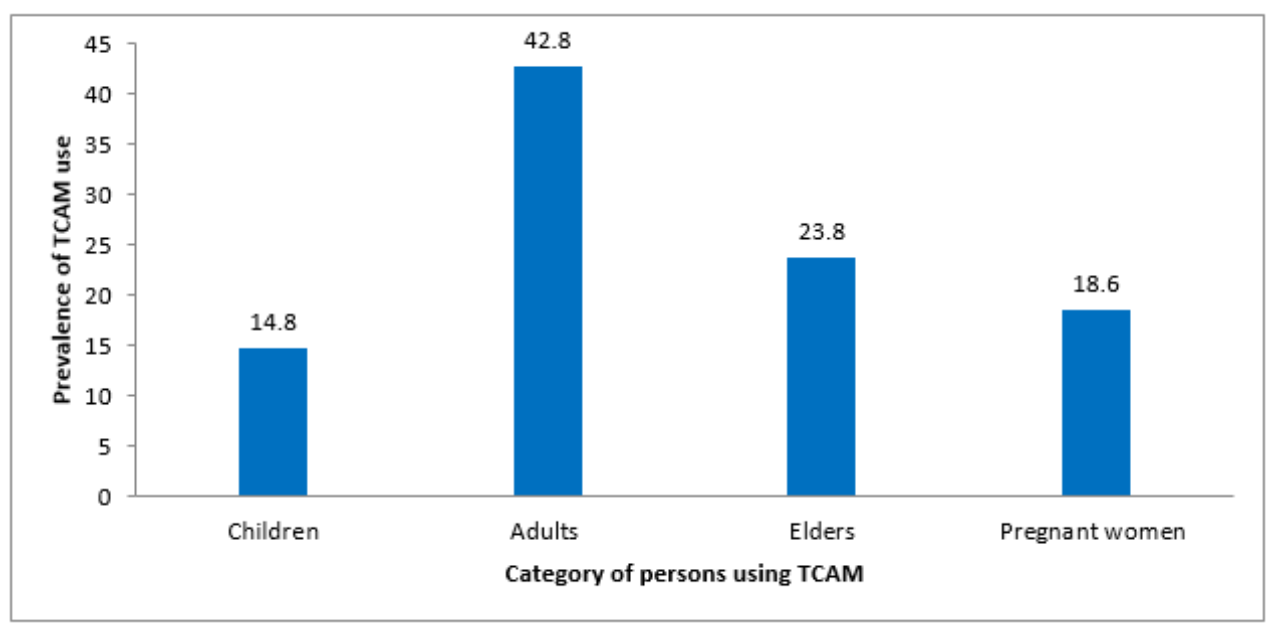

\section{Figure 2}

Category of persons using TCAM in the study communities.

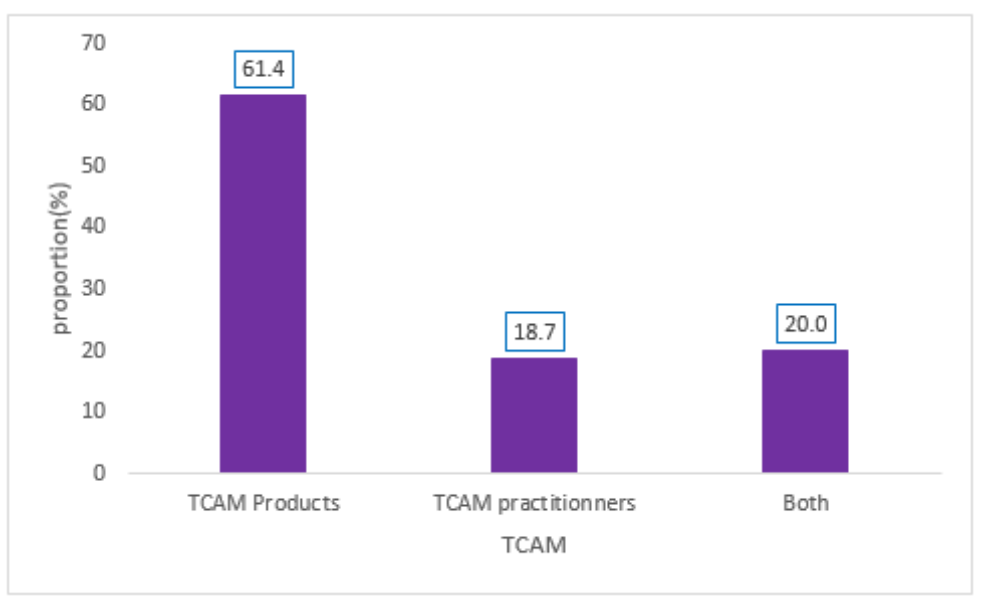

Figure 3

Proportion of community members using TCAM products/practitioner

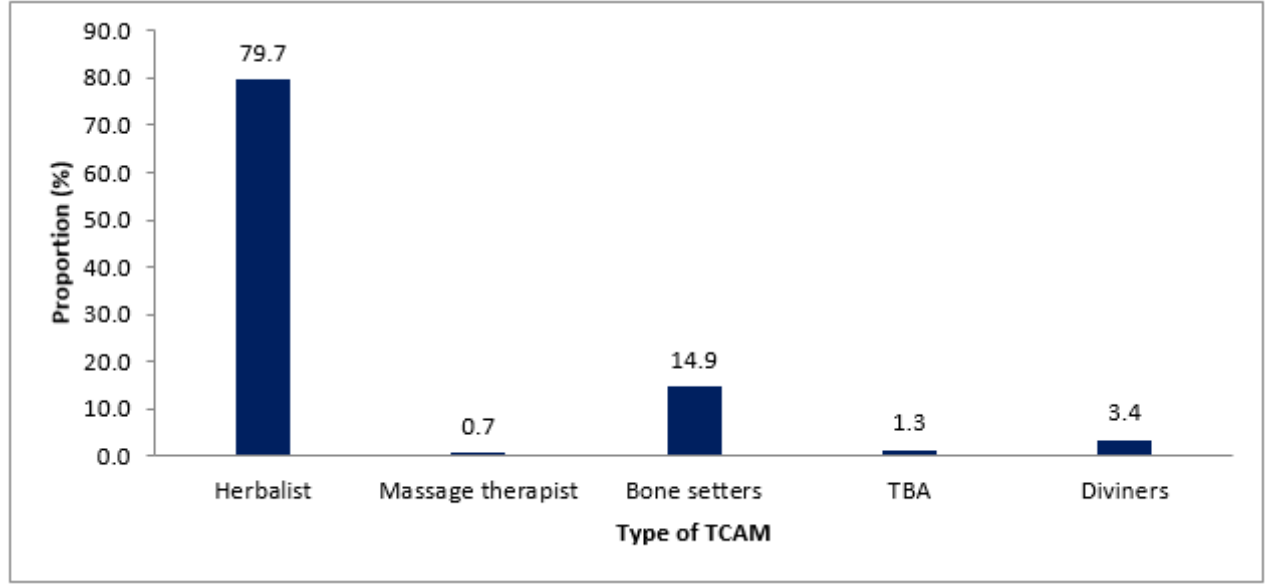

\section{Figure 4}

TCAM practitioners visited by community members 


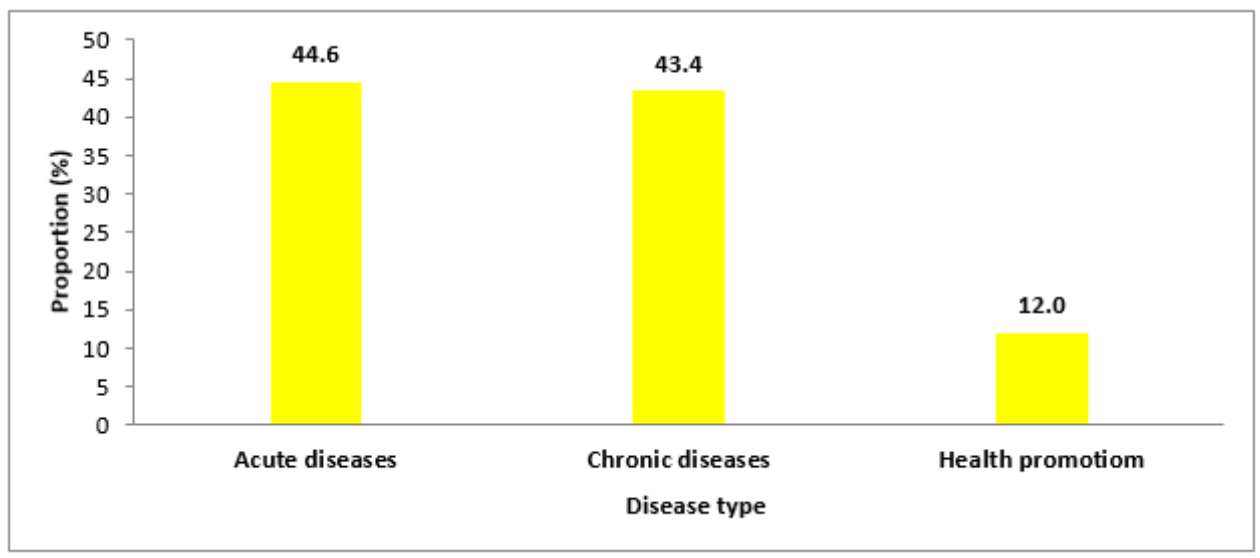

\section{Figure 5}

Types of diseases consulted for by community members

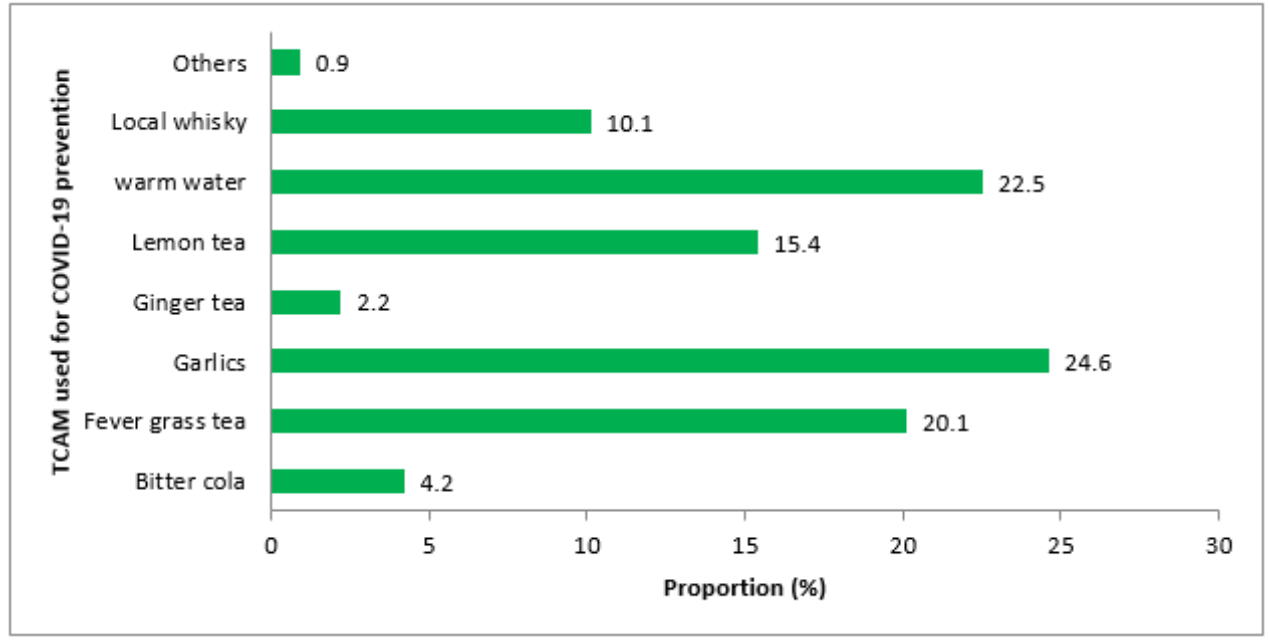

\section{Figure 6}

TCAM products used to prevent COVID-19 